\title{
Close binaries in hierarchical stellar systems
}

\author{
A. Tokovinin \\ Cerro Tololo Interamerican Observatory, (E-mail: atokovinin@ctio.noao.edu)
}

Received: October 22, 2019; Accepted: October 30, 2019

\begin{abstract}
Many (but not all) close binaries are inner pairs in triple and higherorder hierarchical systems. The statistical relation between close binaries and hierarchical systems is well established. Although close binaries can form by a combination of Kozai-Lidov cycles with tides, the study of Moe \& Kratter (2018) indicated that only a fraction of them could be produced by this mechanism and that many close binaries form even before stars contract onto the main sequence. The predicted statistical signatures of tidal formation, such as accumulation of periods just below the tidal cutoff, are not observed. Instead, the main channel of close-binary formation is likely accretion-driven migration. The relation between close binaries and triple stars is not casual, it derives from the common factor which is the accretion strength. High accretion creates massive stars that have a large fraction of close binaries and, at the same time, a large fraction of hierarchical systems.
\end{abstract}

Key words: binaries: close - binaries: multiple

\section{Introduction}

For those who study close binaries (ClBs), the presence of an additional "third" star in the system is an unwanted complication and a nuisance. Yet, it happens so often that many researchers have long suspected some profound relation between ClBs and triple (generally, multiple) systems. Batten (1973) suggested in his book that multiple systems may help us to understand the formation of ClBs that was (and still is) a mystery. Indeed, a nascent star collects gas from a large volume, hence two stars cannot form very close to each other and something must bring them together to make a ClB. Does the third star play a role in this process? Is its presence necessary for making a ClB?

Early data collected from the literature indicated that no less than $40 \%$ of solar-type binaries with $P<10 \mathrm{~d}$ have additional companions, but the frequency of those companions decreases with binary period (Tokovinin, 1997). This finding stimulated further observations that confirmed the relation between ClBs and triples (Tokovinin \& Smekhov, 2002; Rucinski et al., 2007; Tokovinin et al., 2006).

Gravitational interactions between stars in a non-hierarchical multiple system usually lead to ejections. The ejected star removes energy and angular momentum from the system, while the remaining binary hardens. However, the modest decrease in the binary separation by a factor of $3-10$ is insufficient to 
make a $\mathrm{ClB}$, unless the decaying system was itself already very compact. A much more elegant way to form a ClB in a hierarchical triple star was proposed by Eggleton \& Kisseleva-Eggleton (2006). When the mutual orbit inclination exceeds $39^{\circ}$, it oscillates in the Kozai-Lidov cycles, accompanied by the periodic increase in the eccentricity of the inner orbit. The cycles are interrupted if the periastron distance in the inner orbit becomes so small that the tidal friction intervenes and modifies the dynamics of 3 gravitating masses. The Kozai cycles with tidal friction (KCTF) mechanism produces inner binaries with periods below the tidal cutoff ( $P<10 \mathrm{~d}$ for low-mass dwarfs) even in triple systems with wide tertiaries. Assuming randomly oriented orbits, Fabrycky \& Tremaine (2007) predicted that the population of ClBs formed through KCTF has periods $P<10 \mathrm{~d}$, while the mutual inclinations concentrate around $40^{\circ}$ and $140^{\circ}$.

The KCTF mechanism, however, can be responsible for creating only a fraction of ClBs (Moe \& Kratter, 2018). The frequency of ClBs in the pre-main sequence (PMS) and mature stellar populations is similar, while KCTF is too slow to operate at the PMS stage. Moreover, KCTF cannot produce binaries with periods substantially longer than the tidal cutoff. Further insights into the origin of ClBs can be obtained from a detailed study of their statistics.

\section{Solar-type close binaries in multiple systems}

In the 1990s, our knowledge of multiple systems was based on random discoveries, e.g., eclipse timing variations (ETVs) in eclipsing binaries, or visual companions to spectroscopic binaries. Yet, clean, volume-limited and complete samples are needed for statistics. The 25-pc sample of solar-type stars explored by Raghavan et al. (2010) contains only 54 hierarchical systems. The 67-pc sample (Tokovinin, 2014) is $10 \times$ larger, but many binaries discovered by radial velocity (RV) variations or astrometric accelerations have unknown periods.

Several observational programs were conducted to fill the gaps in the 67pc sample. Of relevance here is the spectroscopic survey using the CHIRON echelle spectrometer. Observations of relatively wide visual pairs established that the frequency of subsystems in their primary and secondary components was comparable (Tokovinin, 2015); half of those 96 wide pairs contained a spectroscopic subsystem. A program to determine spectroscopic orbits of stars belonging to multiple systems in the 67-pc sample was started in 2014 and still continues, see references in (Tokovinin, 2019a); counting the Paper VI of these series (Tokovinin, 2019b), the total number of spectroscopic orbits is 45 . With a complementary effort in the northern sky (Gorynya \& Tokovinin, 2018), in the 67-pc sample there remain only 20 inner spectroscopic subsystems with unknown periods, mostly with slow or questionable RV variation.

Figure 1 shows the period-eccentricity relation for inner subsystems with dwarf primaries. The data were extracted from the current version of the multiple- 


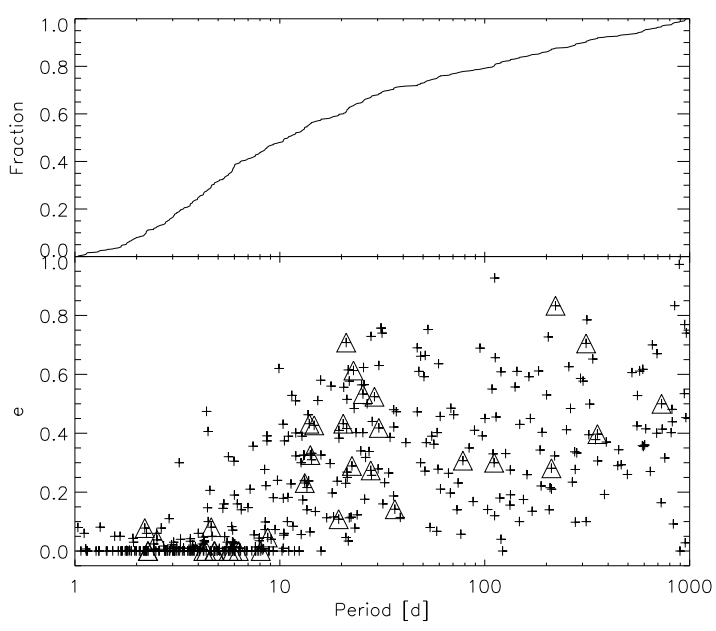

Figure 1. Period-eccentricity relation for inner subsystems of multiple stars from the MSC with masses below 1.5 solar (from Paper VI). Orbits resulting from the $\mathrm{CH}-$ IRON survey are marked by triangles. The upper plot shows the cumulative distribution of periods. The absence of period accumulation just below $10 \mathrm{~d}$ is obvious: the distribution is smooth.

Table 1. Hierarchical multiplicity of close binaries in the 67-pc sample

\begin{tabular}{lcccc}
\hline \hline Period & Total & Solitary & Inner & $P_{\text {out }}<10^{4} \mathrm{~d}$ \\
\hline$<10 \mathrm{~d}$ & 111 & 47 & 64 & 8 \\
$10-100 \mathrm{~d}$ & 121 & 75 & 41 & 7 \\
\hline \hline
\end{tabular}

star catalog, $\mathrm{MSC}^{1}$ (Tokovinin, 2018). Although this data collection is burdened by selection, it should not hide or distort sharp statistical features like the KCTF predicted drop in the number of subsystems with $P>10 \mathrm{~d}$. The circularization is clearly seen in the $P-e$ plot, but the cumulative period distribution is smooth and featureless. A decrease at $P>7 \mathrm{~d}$ noted by Tokovinin \& Smekhov (2002) was a selection effect because most inner subsystems known at that time were discovered by eclipses, with a strong preference of short periods. Nowadays, most subsystems are discovered spectroscopically. The absence of the accumulation of inner periods predicted by KCTF around $P \sim 10 \mathrm{~d}$ in a sample of tight Kepler triples was also noted by Borkovits et al. (2016).

Table 1 shows the statistics of ClBs in the 67-pc sample, updated with all known orbits. At $P<10 \mathrm{~d}$, a fraction $64 / 111=0.58$ of binaries are inner subsystems in known hierarchies; this is a lower limit. For binaries with $10<P<100 \mathrm{~d}$ (incomplete because not all those periods are known), only a fraction $41 / 121=0.34$ are in known triples.

In both period groups, the outer systems are, mostly, wide, while the number of compact triples with $P_{\text {out }}<10^{4} \mathrm{~d}$ is relatively small. However, compact triples, especially those with low-mass tertiaries, are difficult to discover. Accurate accelerations measured by Brandt (2018) by combining the Gaia and

${ }^{1}$ Online access and latest versions at http://www.ctio.noao.edu/ atokovin/stars/index.html 
Hipparcos catalogs reveal 93 stars with significant accelerations among our 232 binaries with $P<100 \mathrm{~d}$. In this group, 62 are already known as multiple, although the accelerations are not necessarily produced by the known wide companions. The remaining 31 systems are potential new compact triples, hence their true number substantially exceeds 15 .

The strong decline of the fraction of triple systems with the increasing period of the inner subsystem, emerging from the statistics in the 67-pc sample, was quantified by Tokovinin et al. (2006). They proved that most binaries with $P>$ $10 \mathrm{~d}$ do not have tertiary companions. This means that most close binaries were formed without the assistance of a tertiary, in agreement with Moe \& Kratter (2018). Another argument against the predominance of the KCTF mechanism is the observed strong tendency for orbit alignment in multiple systems with outer separations below 50 AU (Tokovinin, 2017; Borkovits et al., 2016). Those triples are prime candidates for KCTF, but only a small fraction of them have mutual inclinations $>39^{\circ}$. This said, KCTF must operate in some cases.

\section{Accretion-driven migration}
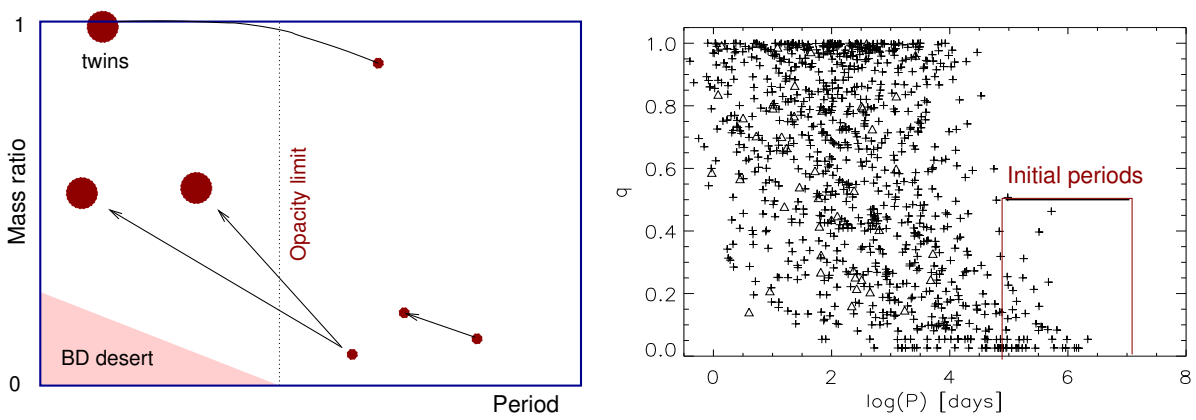

Figure 2. Left: cartoon showing formation and evolution of close binaries by disk fragmentation and accretion. Right: simulated population of solar-type binaries.

An alternative to the KCTF for forming ClBs is migration caused by accretion of gas during the build-up of stellar masses. The orbital angular momentum is extracted by gravitational waves in the circumbinary disks and by ejection of a fraction of the accreted matter. This can be described by

$$
\mathrm{d} a / a=-\eta\left(\mathrm{d} m_{2} / m_{2}\right),
$$

where $\mathrm{d} m_{2}$ is the mass accreted by the secondary component of a binary, $m_{2}$ is its current mass, and $a$ is the orbital separation. The parameter $\eta$ characterizes the speed and direction of migration. The interaction of a binary with accreting 
matter is extremely complex, and there is no general consensus among various studies. Most authors, however, find positive $\eta$ ranging between 1 and 5 (inward migration); see Tang et al. (2017) and references therein.

Figure 2 shows schematically the formation of ClBs. Companions form by fragmentation of a circumbinary disk at large separations $a>40 \mathrm{AU}$ (the socalled opacity limit to fragmentation). Accreted gas is preferentially grabbed by the secondary component and the mass ratio $q$ increases while the orbit shrinks and the total system mass grows. Companions that formed early have initially larger mass ratios and evolve into the $q=1$ regime, producing twin binaries with nearly identical components. Conversely, companions that formed at the end of the mass assembly remain at larger separations and can retain small mass ratios. The brown dwarf desert - the lack of ClBs with brown dwarf companions - does not extend to long periods. Binary evolution in response to accretion depends on many factors and is stochastic; therefore, companions formed with equal initial parameters can end up in binaries with different $P, q$.

Tokovinin \& Moe (2020) developed a mathematical model of accretion-driven migration using simplified prescriptions for the evolution of separation and mass ratio and exploiting its random character. The model successfully reproduces several features of the ClB statistics for both solar-type and massive stars. Massive stars accrete more and, consequently, have a larger multiplicity fraction, a larger fraction of ClBs, and a stronger migration. The distribution of $\log P$ of early B- and O-type binaries grows toward short periods and falls abruptly at $P<0.6 \mathrm{~d}$ corresponding to the contact. This implies that a large fraction of massive binaries have merged during their formation. Merging helps to form very massive stars by delivering part of the final mass in the form of companions. However, strong accretion of gas is still necessary for merging. Assembly of very massive stars from "pre-fabricated" less massive components delays the nuclear evolution time; otherwise, an unrealistically strong accretion would be needed to form a $100 M_{\odot}$ star before it explodes as a supernova.

If ClBs form, mostly, by disk fragmentation and accretion-driven migration, why do so many of them have tertiary components? Moe \& Di Stefano (2017, Fig. 39) noted a tight correlation between the fraction of ClBs and the fraction of triples: both increase with the mass of the primary component. Formation of ClBs and hierarchies is not causally related, but, instead, driven by the common factor, namely the supply of gas during star formation. Strong accretion creates massive stars, and, at the same time, favors formation and migration of inner binaries and formation of further more distant companions from the same accretion flow.

As noted above, compact triple systems tend to be well aligned (nearly coplanar). This is natural if their formation and early evolution was driven by accretion from a common gas reservoir. A class of planetary-like hierarchies is emerging from recent observations. Their orbits are approximately aligned, period ratios are moderate (on the order of 20), and the orbits have small 
eccentricities. A good example is HIP 41431, a compact quadruple system of K7V dwarfs with periods of 2. 9, $59 \mathrm{~d}$, and $3.9 \mathrm{yr}$ (Borkovits et al., 2019).

\section{Summary and outlook}

Recent years have witnessed a substantial progress in our understanding of the formation of ClBs and their relation to hierarchical systems. The decisive factor here are new observational data, especially multiplicity statistics in large and clean samples. The Gaia mission has opened a new era; much is expected from future data releases, especially when the measurements of resolved binaries and radial velocities become available. Large spectroscopic surveys such as APOGEE are also beginning to produce interesting results (Kounkel et al., 2019).

It is very important to extend the multiplicity statistics to very young, PMS populations. If companions formed sequentially by condensing from the accretion disk, young ClBs should not be exactly coeval (Stassun et al., 2008). Observations of interesting young or still forming (Tobin et al., 2016) hierarchical systems will bring new insights and constraints.

The KCTF mechanism of ClB formation is relegated to the second place, while modeling of accretion-driven migration is beginning to give promising results. Much remains to be done in this area, however. ClBs represent only a small fraction of the total binary population. The statistics of most common wide binaries, presumably formed by core fragmentation, still lack plausible theoretical models. The accretion-driven migration is unable to produce $2+2$ hierarchies which, in fact, outnumber the planetary-type $3+1$ quadruple systems (in the 67-pc sample, their ratio is 4:1). Hierarchical systems with misaligned orbits are also quite common, especially with wide separations.

\section{References}

Batten, A. H. 1973, Binary and multiple systems of stars

Borkovits, T., Hajdu, T., Sztakovics, J., et al., A comprehensive study of the Kepler triples via eclipse timing. 2016, Mon. Not. R. Astron. Soc., 455, 4136, DOI: $10.1093 / \mathrm{mnras} / \mathrm{stv} 2530$

Borkovits, T., Sperauskas, J., Tokovinin, A., et al., The compact multiple system HIP 41431. 2019, Mon. Not. R. Astron. Soc., 487, 4631, DOI: 10.1093/mn$\mathrm{ras} / \mathrm{stz} 1510$

Brandt, T. D., The Hipparcos-Gaia Catalog of Accelerations. 2018, Astrophys. J., Suppl., 239, 31, DOI: 10.3847/1538-4365/aaec06

Eggleton, P. P. \& Kisseleva-Eggleton, L., A Mechanism for Producing ShortPeriod Binaries. 2006, Astrophys. Space Sci., 304, 75, DOI: 10.1007/s10509006-9078-z 
Fabrycky, D. \& Tremaine, S., Shrinking Binary and Planetary Orbits by Kozai Cycles with Tidal Friction. 2007, Astrophys. J., 669, 1298, DOI: $10.1086 / 521702$

Gorynya, N. A. \& Tokovinin, A., Spectroscopic orbits of nearby solar-type dwarfs - II. 2018, Mon. Not. R. Astron. Soc., 475, 1375, DOI: 10.1093/mn$\mathrm{ras} / \mathrm{stx} 3272$

Kounkel, M., Covey, K., Moe, M., et al., Close Companions around Young Stars. 2019, Astron. J., 157, 196, DOI: 10.3847/1538-3881/ab13b1

Moe, M. \& Di Stefano, R., Mind Your Ps and Qs: The Interrelation between Period (P) and Mass-ratio (Q) Distributions of Binary Stars. 2017, Astrophys. J., Suppl., 230, 15, DOI: 10.3847/1538-4365/aa6fb6

Moe, M. \& Kratter, K. M., Dynamical Formation of Close Binaries during the Pre-main-sequence Phase. 2018, Astrophys. J., 854, 44, DOI: 10.3847/15384357/aaa6d2

Raghavan, D., McAlister, H. A., Henry, T. J., et al., A Survey of Stellar Families: Multiplicity of Solar-type Stars. 2010, Astrophys. J., Suppl., 190, 1, DOI: 10.1088/0067-0049/190/1/1

Rucinski, S. M., Pribulla, T., \& van Kerkwijk, M. H., Contact Binaries with Additional Components. III. A Search Using Adaptive Optics. 2007, Astron. J., 134, 2353, DOI: 10.1086/523353

Stassun, K. G., Mathieu, R. D., Cargile, P. A., et al., Surprising dissimilarities in a newly formed pair of 'identical twin' stars. 2008, Nature, 453, 1079, DOI: 10.1038/nature07069

Tang, Y., MacFadyen, A., \& Haiman, Z., On the orbital evolution of supermassive black hole binaries with circumbinary accretion discs. 2017, Mon. Not. R. Astron. Soc., 469, 4258, DOI: 10.1093/mnras/stx1130

Tobin, J. J., Kratter, K. M., Persson, M. V., et al., A triple protostar system formed via fragmentation of a gravitationally unstable disk. 2016, Nature, 538, 483, DOI: $10.1038 /$ nature20094

Tokovinin, A., From Binaries to Multiples. I. Data on F and G Dwarfs within 67 pc of the Sun. 2014, Astron. J., 147, 86, DOI: 10.1088/0004-6256/147/4/86

Tokovinin, A., Spectroscopic Subsystems in Nearby Wide Binaries. 2015, Astron. J., 150, 177, DOI: 10.1088/0004-6256/150/6/177

Tokovinin, A., Orbit Alignment in Triple Stars. 2017, Astrophys. J., 844, 103, DOI: $10.3847 / 1538-4357 /$ aa 7746

Tokovinin, A., The Updated Multiple Star Catalog. 2018, Astrophys. J., Suppl., 235, 6, DOI: $10.3847 / 1538-4365 /$ aaala5

Tokovinin, A., Spectroscopic Orbits of Subsystems in Multiple Stars. V. 2019a, Astron. J., 157, 91, DOI: 10.3847/1538-3881/aafe00 
Tokovinin, A., Spectroscopic Orbits of Subsystems in Multiple Stars. VI. 2019b, Astron. J., 158, 222, DOI: 10.3847/1538-3881/ab4c94

Tokovinin, A. \& Moe, M., Formation of close binaries by disc fragmentation and migration, and its statistical modelling. 2020, Mon. Not. R. Astron. Soc., 491, 5158, DOI: $10.1093 / \mathrm{mnras} / \mathrm{stz} 3299$

Tokovinin, A., Thomas, S., Sterzik, M., \& Udry, S., Tertiary companions to close spectroscopic binaries. 2006, Astron. Astrophys., 450, 681, DOI: 10.1051/0004-6361:20054427

Tokovinin, A. A., On the multiplicity of spectroscopic binary stars. 1997, Astronomy Letters, 23, 727

Tokovinin, A. A. \& Smekhov, M. G., Statistics of spectroscopic sub-systems in visual multiple stars. 2002, Astron. Astrophys., 382, 118, DOI: 10.1051/00046361:20011586 